\title{
Autologous Graft-Versus-Host Disease of the Gastrointestinal Tract in Patients With Multiple Myeloma and Hematopoietic Stem Cell Transplantation
}

\author{
Muhammad B. Hammami ${ }^{\mathrm{a}, \mathrm{f}}$, Rebecca Talkin ${ }^{\mathrm{b}}$, Ahmad M. Al-Taeec, \\ Martin W. Schoen ${ }^{\text {, }}$, Sagun D. Goyal ${ }^{\mathrm{d}}$, Jin-Ping Lai ${ }^{\mathrm{e}}$
}

\begin{abstract}
Multiple myeloma (MM) is the most common indication for autologous hematopoietic stem cell transplantation (HSCT) in North America. Despite occurring in up to $50 \%$ of patients undergoing allogeneic HSCT, the incidence of graft-versus-host disease (GVHD) after autologous HSCT is reportedly only $5-20 \%$. Gastrointestinal involvement with graft-versus-host disease (GI GVHD) is a common and serious complication of allogeneic HSCT. GI GVHD after autologous transplant, which is referred to as autologous GVHD (auto-GVHD), has also been described. Auto-GVHD is usually less severe than allogeneic GVHD, and it can be one of the manifestations of engraftment syndrome with release of inflammatory cytokines and infiltration of auto-reactive $\mathrm{T}$ cells into affected tissue. Seventy-nine percent of patients respond well to corticosteroids without evidence of recurrence. However, cases of severe auto-GVHD lacking good response to corticosteroids have been reported, most notably in MM patients. Here we present two cases of autologous GI GVHD in recipients of autologous HSCT for treatment of MM. Our cases demonstrate two distinct clinical and endoscopic presentations of this uncommon entity. In the first case, the patient had more severe clinical symptoms accompanied by radiographic, endoscopic, and pathologic findings. The hospital course was complicated by cryptosporidium enteritis and acute cholecystitis in the setting of increased immunosuppression with a corticosteroid for presumed auto-GVHD. In contrast, the second case presented a patient with normal radiologic and endoscopic findings. Pathology revealing frequent apoptotic bodies led to auto-
\end{abstract}

Manuscript submitted October 23, 2017, accepted November 15, 2017

${ }^{a}$ Division of Gastroenterology, Department of Internal Medicine, School of Medicine, Saint Louis University, St. Louis, MO, USA

${ }^{b}$ School of Medicine, Saint Louis University, St. Louis, MO, USA

'Department of Internal Medicine, School of Medicine, Saint Louis University, St. Louis, MO, USA

${ }^{\mathrm{d}}$ Division of Hematology, Oncology \& Cellular Therapy, Department of Internal Medicine, School of Medicine, Saint Louis University, St. Louis, MO, USA

eDepartment of Pathology, University of Florida, Gainesville, FL, USA

${ }^{\mathrm{f} C}$ Corresponding Author: Muhammad B. Hammami, Division of Gastroenterology and Hepatology, Department of Internal Medicine, Saint Louis University School of Medicine, 1402 S. Grand Blvd., St Louis, MO 63104, USA.

Email: mbhammami@hotmail.com

doi: https://doi.org/10.14740/gr925w
GVHD as a diagnosis. Both our patients received similar courses of chemotherapy prior to autologous HSCT (four cycles of a proteasome inhibitor, lenalidomide, and dexamethasone). Our work highlights the importance of maintaining a high level of clinical suspicion for auto-GVHD in patients presenting with GI symptoms after autologous HSCT, as it is a potentially treatable pathology that may be easily confused with other conditions. Health care providers should be aware of the potential complications of auto-GVHD after autologous HSCT and should be suspicious of auto-GVHD if GI symptoms occur, especially in patients receiving immunomodulatory therapy for $\mathrm{MM}$, even in the absence of gross endoscopic findings.

Keywords: Multiple myeloma; Autologous hematopoietic stems cell transplant; Graft versus host disease

\section{Introduction}

Multiple myeloma (MM) is the most common indication for autologous hematopoietic stem cell transplantation (HSCT) in North America [1]. Despite occurring in up to $50 \%$ of patients undergoing allogeneic HSCT, the incidence of graft-versushost disease (GVHD) after autologous HSCT is reportedly only $5-20 \%$ [2]. Gastrointestinal involvement with graft-versus-host disease (GI GVHD) is a common and serious complication of allogeneic HSCT. GI GVHD after autologous transplant, which is referred to as autologous GVHD (autoGVHD), has also been described [1].

Several hypotheses have been proposed to explain the pathophysiology of auto-GVHD, but none have been proven [1]. Auto-GVHD is usually less severe than allogeneic GVHD, and it can be one of the manifestations of engraftment syndrome with release of inflammatory cytokines and infiltration of auto-reactive T cells into affected tissue [3]. Seventy-nine percent of patients respond well to corticosteroids without evidence of recurrence [4]. However, cases of severe auto-GVHD lacking good response to corticosteroids have been reported, most notably in MM patients [5]. Here we present two cases of autologous GI GVHD in recipients of autologous HSCT for treatment of MM. Our cases demonstrate two distinct clinical and endoscopic presentations of autologous GI GVHD. Our work highlights the importance of maintaining a high level of clinical suspicion for auto-GVHD in patients presenting with 


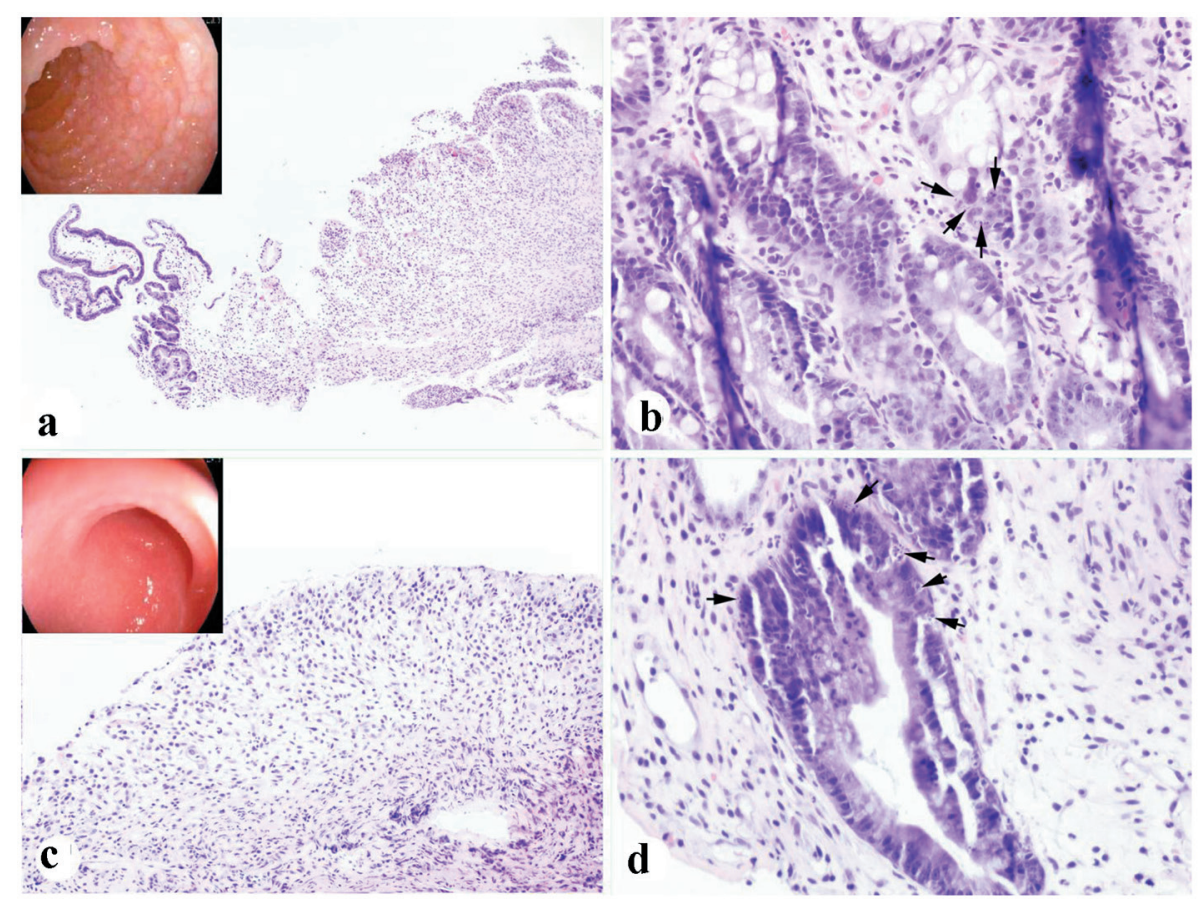

Figure 1. Severe duodenal and colonic auto-GVHD present in case 1. (a, b) Duodenal biopsy showing focal mucosal sloughing, crypt injury and dropout, and epithelial apoptotic bodies (arrows) (inset in a: endoscopic appearance). (c, d) Colonic biopsy showing extensive mucosal sloughing, crypt injury and dropout, and epithelial apoptotic bodies (arrows) (inset in c: endoscopic appearance) (H\&E stain. a, c: × 100; b, d: × 400).

GI symptoms after autologous HSCT, as it is a potentially treatable pathology that may be easily confused with other conditions.

\section{Case Reports}

\section{Case 1}

A 63-year-old man with MM achieved complete remission following four cycles of carfilzomib, lenalidomide, and dexamethasone and later underwent autologous HSCT after highdose melphalan. He had little regimen-related GI toxicity during his count nadir. Post-engraftment, at day +20 following transplantation, he was admitted from the bone marrow transplant clinic with a 2-day history of intermittent nausea, vomiting, and diarrhea, as well as poor appetite and weight loss.

Vital signs were notable for mild tachycardia. Physical examination revealed dry mucous membranes, bilateral pedal edema, and normoactive bowel sounds with no evidence of abdominal distention or tenderness. Laboratory testing was remarkable for a white blood cell count of $1.9 \times 10^{3} / \mu \mathrm{L}$ with an absolute neutrophil count of $0.7 \times 10^{3} / \mu \mathrm{L}$, sodium of 135 $\mathrm{mmol} / \mathrm{L}$, potassium of $3.2 \mathrm{mmol} / \mathrm{L}$, and albumin of $2.1 \mathrm{~g} / \mathrm{dL}$. Stool studies including Clostridium difficile toxin assay, giardia and cryptosporidial antigens, ova and parasite examination, and stool cultures were all negative. Contrasted computed tomography of the abdomen and pelvis demonstrated diffusely thickened and edematous walls of the stomach, small bowel, and colon, suggestive of diffuse gastritis and enterocolitis. Since his admission, he was noted to be hypotensive and tachycardic but afebrile on a number of occasions, so a one-time dose of amikacin was administered. Total parenteral nutrition was initiated, as he did not tolerate enteral feeding.

Given radiological evidence of GI involvement and persistent symptoms, endoscopic evaluation was performed on day 24 from HSCT. Esophagogastroduodenoscopy (EGD) demonstrated severe duodenopathy (Fig. 1a). Duodenal biopsies revealed evidence of ulcer formation, surface epithelial sloughing, and frequent epithelial apoptotic bodies in the crypts (Fig. $1 \mathrm{a}, \mathrm{b})$. Colonoscopy was notable for edematous mucosa in the rectum, sigmoid and descending colon. There was extensive mucosal ulceration with loss of vascular markings throughout the left colon (Fig. 1c). Random colonic biopsies obtained revealed surface epithelial sloughing, crypt injury and dropout, and numerous epithelial apoptotic bodies present in the residual crypts (Fig. 1c, d). Active inflammation was very mild, and there was no evidence of pathologic surface organisms, viral inclusions, or plasmacytic aggregates on microscopic examination. Methylprednisolone $1 \mathrm{mg} / \mathrm{kg}$ intravenous (IV) daily was started for GVHD of the GI tract.

Due to lack of response to methylprednisolone $1 \mathrm{mg} / \mathrm{kg}$ daily, the dose was increased to $1 \mathrm{mg} / \mathrm{kg}$ twice daily, and the patient underwent repeat endoscopic evaluation $($ day +36$)$. The colonoscopy now demonstrated patchy, congested and nodular mucosa with multiple superficial 6 - $10 \mathrm{~mm}$ ulcers in the transverse, descending, and sigmoid colon (Fig. 2a). Colonic biopsies revealed crypt regeneration and frequent epithelial apoptotic bodies (Fig. 2a, b). The EGD demonstrated nodular mu- 


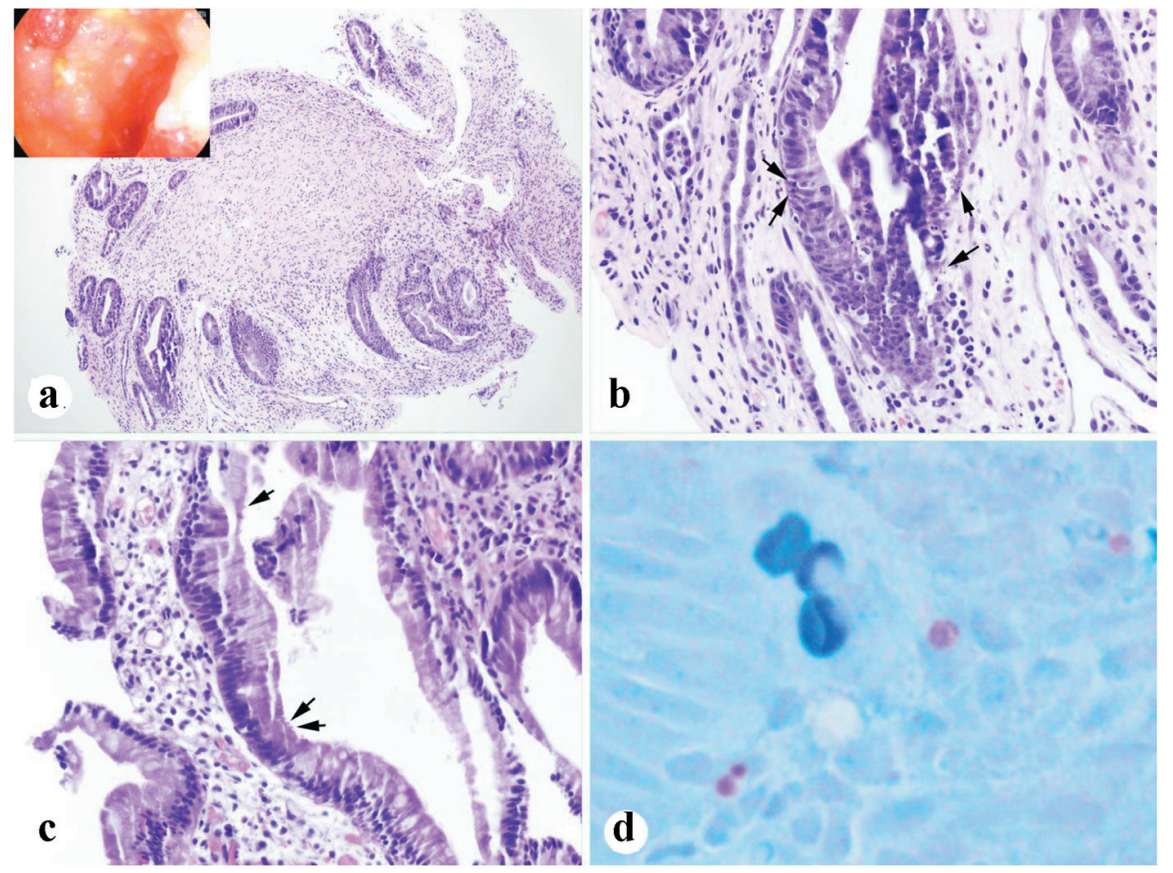

Figure 2. Biopsies after treatment for 13 days. (a, b) Colonic biopsy showing crypt regeneration and epithelial apoptotic bodies (arrows) (inset in a: endoscopic appearance). (c, d) Duodenal biopsy showing rare organisms of cryptosporidium (arrows) and highlighted by AFB (acid-fast) stain (d) (a: × 100; b, c: $\times 400$; d: original, $\times 600)$.

cosa in the entire examined duodenum, as well as non-bleeding gastric erosions. Duodenal and gastric biopsies revealed few apoptotic bodies consistent with GVHD-like disease, as well as few organisms suspicious for cryptosporidium (Fig. 2c, d). Immuno-histochemical stains for CMV were negative.

The patient required a prolonged course of oral prednisone and TPN for management of auto-GVHD of the GI tract. His hospital course was complicated by acute cholecystitis and atrial fibrillation with rapid ventricular response. Moreover, despite having three negative cryptosporidium stool antigen tests, previous findings on duodenal biopsy were concerning for an active cryptosporidial infection, so a 10-day course of nitazoxanide was completed. Over the course of the next 2 months of his hospitalization, the GI symptoms improved, he was able to tolerate an oral diet and was eventually released home. One month later, he presented with loss of appetite, weight loss, and hypoalbuminemia. He was restarted on TPN, which improved his symptoms and was later discontinued after 4 months. Over the following 19 months, and upon subsequent clinic visits, his appetite and energy levels continued to gradually improve without recurrence of symptoms. Because of the severity of his GI disease, maintenance therapy with immunomodulatory agents was not started post-transplant.

\section{Case 2}

A 74-year-old man with a history of MM completed four cycles of chemotherapy with lenalidomide, bortezomib, and dexamethasone with partial response. His treatment was consolidated 4 months later with autologous HSCT and high-dose melphalan for conditioning.

Within the following 2 weeks, he developed abdominal distention and progressively worsening diarrhea. Vital signs were within normal values. Physical exam revealed dry mucous membranes and mild non-localized abdominal tenderness with deep palpation. Laboratory testing revealed a white blood cell count of $3.1 \times 10^{3} / \mu \mathrm{L}$, with an absolute neutrophil count of $2.3 \times 10^{3} / \mu \mathrm{L}$, along with normal liver enzymes, blood creatinine, and electrolytes. Stool studies and cultures were negative for an infectious etiology to explain the diarrhea. Abdominal imaging was consistent with an ileus that resolved with supportive measures. Further evaluation with a flexible sigmoidoscopy revealed grossly normal appearing colonic mucosa (Fig. 3a). However, colonic biopsies demonstrated frequent apoptotic bodies consistent with acute autologous GVHD (Fig. 3b). No viral inclusions were identified and cytomegalovirus immunostain was negative. A 6-day course of high-dose intravenous steroids was initiated with complete resolution of symptoms. He was discharged home with a 4-week taper of oral steroids, with no recurrence of symptoms on further outpatient follow-up. Because of high-risk myeloma, maintenance therapy was given post-transplant, but immunomodulatory therapy and proteasome inhibitor therapy were added sequentially to ensure that they did not result in a flare of GVHD.

\section{Discussion}

Several hypotheses have been proposed to explain the pathophysiology of GVHD that occurs after autologous HSCT in $5-20 \%$ of patients [2]. These include and are not limited to: al- 


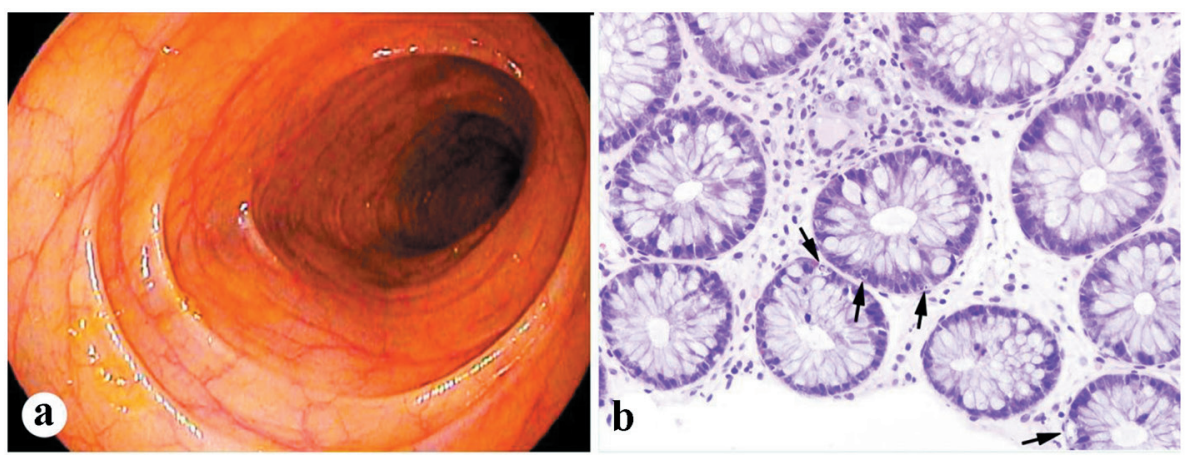

Figure 3. Mild colonic GVHD present in case 2. (a) Unremarkable endoscopic appearance of the colon. (b) Frequent apoptotic bodies present in the crypts of the colonic biopsy (arrows, H\&E stain, $\times 400$ ).

teration of the immune system composition due to an underlying pathology such as MM, and the use of immunomodulatory medications as part of the conditioning regimen for autologous HSCT [1]. Others have postulated that the use of granulocytecolony stimulating factors (G-CSF) mobilizes peripheral blood stem cell grafts, resulting in a loss of self-tolerance and autoimmune disease [5]. These conditions can result in the creation of auto-reactive $\mathrm{T}$ cells and the suppression of $\mathrm{T}$ regulatory cells, leading to inflammation and immune infiltrates in affected tissue [3].

In a study by Holmberg et al involving 681 patients who received autologous HSCT, the reported incidence of autoGVHD involving the GI tract was $13 \%$. The mean time to developing GI symptoms was 15 days after autologous HSCT. The most common presenting symptoms were persistent nausea and vomiting (90\%), followed by diarrhea (40\%) [4]. Other reported symptoms included anorexia, dysphagia, and abdominal pain [4]. The differential diagnosis of GI symptoms following autologous HSCT is very broad. This differential diagnosis includes and is not limited to: medication side effects, neutropenic enterocolitis, infectious colitis, engraftment syndrome, IBS, hormonal disturbances, GI amyloidosis, and exacerbation of preexisting pathology [6]. The diagnosis of autologous GI GVHD can be made on the basis of ongoing GI symptoms, endoscopic evidence of mucosal abnormalities, and histological findings of apoptotic epithelial cells or crypt cell dropout with lymphocytic infiltrate [4].

Here we presented two cases of GI GVHD in recipients of autologous HSCT for treatment of MM. Our cases demonstrate two distinct clinical and endoscopic presentations of this uncommon entity. In the first case, the patient had more severe clinical symptoms (chronic diarrhea and weight loss) accompanied by radiographic, endoscopic, and pathologic findings. The hospital course was complicated by Cryptosporidium enteritis and acute cholecystitis in the setting of increased immunosuppression with a corticosteroid for presumed auto-GVHD. In contrast, the second case presented a patient with normal radiologic and endoscopic findings. Pathology revealing frequent apoptotic bodies led to auto-GVHD as a diagnosis. Both our patients received similar courses of chemotherapy prior to autologous HSCT (four cycles of a proteasome inhibitor, lenalidomide, and dexamethasone). These immunomodulatory therapies may have contributed to the development of GI
GVHD. However, each patient displayed distinct symptoms, gross/microscopic findings, and response to therapy. While the first patient had a slower response to IV methylprednisolone and required months of TPN to reach full recovery, the second patient responded quickly to IV steroids with complete resolution of symptoms within days. Therefore, physicians, and specifically gastroenterologists, should have a low threshold to obtain biopsies after autologous HSCT if the clinical presentation is suggestive of GVHD, even in patients with grossly normal appearing mucosa. Prompt diagnosis and subsequent treatment may help alleviate symptoms and prevent the progression to a more severe disease or chronic GVHD.

High clinical suspicion for the development of autoGVHD is especially important in MM patients, who may be at a greater risk for developing auto-GVHD [7]. In a study by Batra et al, five cases of auto-GVHD were reported among $223 \mathrm{MM}$ patients. On the other hand, none of the 136 patients who underwent autologous HSCT transplantation for Hodgkin disease, acute myeloid leukemia, or non-Hodgkin lymphoma developed this complication [7]. Additionally, there have been reports that suggest that auto-GVHD, particularly in MM patients, can follow a more severe course and is not responsive to steroids. A study by Drobyski et al of 386 patients undergoing autologous HSCT reported five MM patients who experienced severe auto-GVHD manifesting as skin rash and profuse diarrhea. These cases of auto-GVHD were not responsive, or were only partially responsive to corticosteroids, resulting in the fatality of three patients [5]. It is notable that these more severe cases of auto-GVHD occurred only in MM patients and not in any other hematological malignancies included in the study [5, 8]. In another fatal case of auto-GVHD following autologous HSCT in an MM patient, the patient was discharged without steroids, as her symptoms of fever and diarrhea were attributed to a less severe form of engraftment syndrome, which overlaps with auto-GVHD [8]. Engraftment syndrome is a broader term which includes a continuum of complications following autologous HSCT with a spectrum of disease severities. Complications of engraftment syndrome may include rash, fever, capillary leak, encephalopathy, diarrhea, and renal and hepatic dysfunction. Auto-GVHD of the GI tract often presents on the more severe end of the spectrum of engraftment syndrome but may be missed without a tissue biopsy confirming GVHD pathology [3]. 
These cases highlight the importance of careful and prolonged monitoring of patients for symptoms of auto-GVHD following autologous HSCT, especially in MM patients. MM patients may experience a higher incidence, and potentially more severe course of auto-GVHD, which helps to support the hypothesis that a significant contributor to the development of auto-GVHD may be alterations of the immune system by the disease itself. This increased frequency and severity may also be due to the immunomodulatory therapies, such as lenalidomide (Revlimid) and pomalidomide (Pomalyst), used in the treatment of MM. These drugs inhibit the proliferation and function of regulatory $T$ cells, which may then be incapable of regulating the immune response in those receiving autologous HSCT [9]. While immunomodulatory therapies are effective at preventing relapse in MM patients, patients on lenalidomide who develop persistent GI symptoms should prompt endoscopic evaluation and biopsy.

Furthermore, our two patients show a range of outcomes with auto-GVHD. In the second patient, a single course of steroids was sufficient to reverse the symptoms of auto-GVHD. However, the first patient had a more severe disease course complicated by possible Cryptosporidium enteritis and ulcers evident on endoscopy. This patient appears to have developed steroid-resistant disease and a clinical scenario similar to inflammatory bowel disease. Interestingly, some data suggest that anti-tumor necrosis alpha therapy in the treatment of GIGVHD can be helpful and may play a role in future treatment of auto-GVHD [10].

\section{Conclusion}

Case reports such as ours are important to further the understanding of the various presentations of auto-GVHD, the potential contributing factors that may predispose patients to auto-GVHD, and to highlight effective treatment approaches. Healthcare providers should be aware of the potential complications of auto-GVHD after autologous HSCT, be suspicious of GVHD if GI symptoms occur, and should consider obtaining biopsies from the GI tract even in the absence of gross endoscopic findings, especially in patients receiving immunomodulatory therapy for MM.

\section{Funding}

None.

\section{Conflict of Interest}

All authors have no conflict of interest to declare.

\section{Ethical Approval}

This article does not contain any studies with human participants or animals performed by any of the authors.

\section{Consent}

Informed consent was obtained from all individual participants included in the study.

\section{Author Certification}

All authors certify that they have participated sufficiently in the intellectual content of this paper. Each author has reviewed the final version of the manuscript and approves it for publication. Should the editors request the data upon which the work is based, the authors shall produce it.

\section{References}

1. Lazarus HM, Sommers SR, Arfons LM, Fu P, Ataergin SA, Kaye NM, Liu F, et al. Spontaneous autologous graft-versus-host disease in plasma cell myeloma autograft recipients: flow cytometric analysis of hematopoietic progenitor cell grafts. Biol Blood Marrow Transplant. 2011;17(7):970-978.

2. Goddard DS, Ruben BS, Mathes ED, Nixon M, Wolf J, Fox LP. A case of severe cutaneous, GI and liver GVHD in a patient with multiple myeloma, status-post-second auto-SCT. Bone Marrow Transplant. 2010;45(2):409-411.

3. Cornell RF, Hari P, Drobyski WR. Engraftment syndrome after autologous stem cell transplantation: an update unifying the definition and management approach. Biol Blood Marrow Transplant. 2015;21(12):2061-2068.

4. Holmberg L, Kikuchi K, Gooley TA, Adams KM, Hockenbery DM, Flowers ME, Schoch HG, et al. Gastrointestinal graft-versus-host disease in recipients of autologous hematopoietic stem cells: incidence, risk factors, and outcome. Biol Blood Marrow Transplant. 2006;12(2):226234.

5. Drobyski WR, Hari P, Keever-Taylor C, Komorowski R, Grossman W. Severe autologous GVHD after hematopoietic progenitor cell transplantation for multiple myeloma. Bone Marrow Transplant. 2009;43(2):169-177.

6. Krishna SG, Barlogie B, Lamps LW, Krishna K, Aduli F, Anaissie E. Recurrent spontaneous gastrointestinal graftversus-host disease in autologous hematopoietic stem cell transplantation. Clin Lymphoma Myeloma Leuk. 2010;10(1):E17-21.

7. Batra A, Cottler-Fox M, Harville T, Rhodes-Clark BS, Makhoul I, Nakagawa M. Autologous Graft versus Host Disease: An Emerging Complication in Patients with Multiple Myeloma. Bone Marrow Res. 2014;2014:891427.

8. Fidler C, Klumpp T, Mangan K, Martin M, Sharma M, Emmons R, Lu M, et al. Spontaneous graft versus host disease occurring in a patient with multiple myeloma after autologous stem cell transplant. Am J Hematol. 2012;87(2):219-221.

9. Galustian C, Meyer B, Labarthe MC, Dredge K, Klaschka D, Henry J, Todryk S, et al. The anti-cancer agents lenalidomide and pomalidomide inhibit the proliferation 
and function of T regulatory cells. Cancer Immunol Immunother. 2009;58(7):1033-1045.

10. Levine JE, Paczesny S, Mineishi S, Braun T, Choi SW,
Hutchinson RJ, Jones D, et al. Etanercept plus methylprednisolone as initial therapy for acute graft-versus-host disease. Blood. 2008;111(4):2470-2475. 